\title{
Efficacité du Biostimulant Banzaï TM sur l'amélioration de la couverture foliaire des cacaoyers apparemment sains et des cacaoyers atteints de la maladie du "Swollen shoot" à Petit Bondoukou, Sud-Ouest de la Côte d'Ivoire
}

\author{
Franck Zokou Oro*1, Hermann-Desiré Lallie², Guy Kouassi Brou', Pacôme Bi-Zaouli, ${ }^{1}$ Jesus Inza Fofana ${ }^{2}$, \\ and Hortense Atta Diallo ${ }^{3}$ \\ 1Département de biologie végétale, Unité de Formation et de Recherche (UFR) des Sciences Biologiques, Université \\ Peleforo GON COULIBALY. BP 1328 Korhogo \\ 2Département de biochimie-génétique, Unité de Formation et de Recherche (UFR) des Sciences Biologiques, \\ Université Peleforo GON COULIBALY. BP 1328 Korhogo. \\ 3Unité de Recherche en Phytopathologie, Département de Protection de Végétaux et de l'Environnement, Université \\ Nangui Abrogoua. \\ *Corresponding author: franckoro@yahoo.fr (00225 5886 15 10)
}

Original submitted in on $3^{\text {rd }}$ December 2019. Published online at www.m.elewa.org/journals/ on 29th February 2020 https://doi.org/10.35759/JABs.v146.9

\section{RÉSUMÉ}

Objectifs: Cette étude a consisté a consisté à évaluer l'efficacité du Biostimulant «BanzaïTM » sur l'amélioration de la couverture foliaire des cacaoyers sains et affectés par le CSSV à Soubré. II s'agit spécifiquement de comparer l'effet de la simple dose et de la double dose de Banzaï'M sur le développement foliaire du cacaoyer.

Méthodologie et résultats : Le dispositif expérimental, répété quatre fois, est constitué d'un bloc de quatre parcelles avec chacune dix arbres malades et dix arbres sains notées T0 (Témoin), T1 (une application de Banzaï ${ }^{T M}$ à dose normale sans engrais), T2 (une application de Banzai ${ }^{T M}$ à double dose sans engrais) et T3 (une application de Banzaï ${ }^{\mathrm{TM}}$ à dose normale avec engrais). Le produit a été appliqué durant quatre mois et les observations ont été faites sur six mois. Les résultats obtenus ont montré que BanzaïTM améliore significativement la couverture foliaire des cacaoyers malades et sains indépendamment de la dose du biostimulant et de l'apport d'engrais.

Conclusion et application des résultats : Cette étude a montré que l'application du biostimulant a eu un impact significativement positif sur la couverture foliaire des cacaoyers sains et des malades. Dans le cas des cacaoyers sains, la parcelle T1 à simple dose de BanzaïTM sans engrais a été plus efficace que la double dose sans engrais (T2), contrairement aux cacaoyers malades chez lesquels la double dose a eu plus d'effet escompté. Les résultats obtenus dans cette étude pourraient permettre aux producteurs de cacaoyers d'adopter de nouvelles techniques de lutte culturale contre le virus du "Swollen shoot". En effet, pour une parcelle atteinte, il serait recommandable de détruire les arbres malades et maintenir les arbres apparemment sains, sur lesquels le biostimulant pourrait être appliqué pour optimiser la production. Cela contrarie les méthodes actuelles de lutte culturale qui consistent à détruire et les arbres malades et les arbres sains dans un rayon bien déterminé autour du foyer.

Mots clés : Cacaoyer, Biostimulant, Banzaï ${ }^{T M}$, CSSV, Côte d'Ivoire, Soubré. 


\section{ABSTRACT}

Objectives: This study consisted in evaluating the effectiveness of Biostimulant "BanzaïTM" on improvement of the foliar cover of healthy cocoa trees and affected trees by the CSSV disease in Soubré. Specifically, the effect of single dose and double dose of Banzaï ${ }^{\mathrm{TM}}$ on foliar cover development of cocoa trees is compared.

Methodology and results: The experimental device, repeated four times, consists of a block of four plots with ten diseased trees and ten healthy trees noted T0 (control), T1 (normal dose of BanzaiTM without fertilizer), T2 (two-dose of BanzaïTM without fertilizer) and T3 (normal-dose of Banzaï ${ }^{\mathrm{TM}}$ with fertilizer). Banzai TM product was applied for four months and observations were made over six months. The results obtained showed that Banzaï ${ }^{\mathrm{TM}}$ significantly improves the foliar cover of diseased and healthy cocoa trees regardless of the dose of the biostimulant and fertilizer.

Conclusion and application of the results: This study showed that the application of the biostimulant had a significantly positive impact on the foliar cover of healthy cocoa trees and disease trees. In the case of healthy cocoa trees, the single-dose T1 (plot of BanzaïTM without fertilizer) was more effective than the double dose without fertilizer (T2), in contrast to the diseased cocoa trees in which the double dose had more expected effect. The results obtained in this study could enable cocoa farmers to adopt new cultural control technics against Swollen shoot virus. Indeed, for an affected plot, it would be advisable to destroy diseased trees and maintain apparently healthy trees, on which the biostimulant could be applied to optimize production. This is contrary to current cultural control methods of destroying diseased trees and healthy trees within a specific radius around the outbreaks.

Keywords : Cocoa trees, foliar cover, Biostimulant, BanzaïTM, CSSV, Côte d'Ivoire.

\section{INTRODUCTION}

La Côte d'Ivoire est un pays tropical situé en Afrique de l'Ouest dont l'économie est basée sur l'agriculture. Le cacao occupe une place de choix dans le tissu socio-économique de la Côte d'Ivoire qui en produit annuellement environ 165000 tonnes, soit $32 \%$ de l'offre mondiale, faisant de ce pays le premier producteur mondial (JNCC, 2016). La culture du cacao génère $35 \%$ des recettes totales d'exportation du pays et représente $15 \%$ du PIB et 20\% des recettes fiscales (Dufumier, 2016). Cependant, la culture du cacao est mise à mal par plusieurs contraintes dont l'existence de ravageurs et de maladies telle que le Swollen shoot qui a une influence négative sur les rendements et la qualité des fèves de cacao. En effet, le "Swollen shoot" est une maladie virale du cacaoyer causé par un virus du genre Badnavirus de la famille des Caulimoviridea (Muller et Sackey, 2005). La première forme du virus fut détectée au Ghana en 1936 sur des jeunes cacaoyers de quinze ans (Lot et al., 1991). Le "Swollen shoot" a été par la suite signalé dans d'autre pays producteurs de cacao comme la Côte d'Ivoire en 1946, le Nigeria en 1944 et le Togo en 1955 (Alibert, 1946). La transmission naturelle du virus CSSV d'une plante malade à une plante saine se fait par les cochenilles farineuses (Posnette, 1940) de la famille des Pseudococcidae et de manière semi persistante (Posnette, 1940 ; Ollennu et al., 1989). Le CSSV une fois dans la plante, provoque des perturbations physiologiques et morphologiques (Van, 1998). Le virus provoque un gonflement suivi de l'avortement de la racine pivotante, l'avortement des apex des rameaux et l'inhibition des fonctionnements du méristème terminal suivi d'un ralentissement de la croissance de l'arbre (Oro, 2011). Les organes de reproduction (grains de pollen et les ovules) deviennent anormaux et stériles. Les cabosses malades sont anormales et les feuilles des arbres atteints deviennent gaufrées (Kouakou et al, 2014). Le virus provoque des décolorations plus ou moins intenses du limbe suivant son degré de sévérité. Le virus occasionne une faible floraison, un faible taux de nouaison, un dessèchement physiologique très prononcé et une diminution rapide du nombre de cabosses produites (Van, 1998). Dans les foyers, une défoliation progressive des cacaoyers est 
observée provoquant ainsi un trou de lumière. II s'en suit alors l'apparition des mauvaises herbes et le desséchement des branches (Kébé et al., 2016). Cette maladie provoque aussi des rougeurs sur de jeunes feuilles (Figure $1 \mathrm{~A}$ ), et des gonflements sur les jeunes tiges (Figure 1B) entrainant ainsi la mort de l'arbre au bout de cinq ans (Figure $1 \mathrm{C}$ ) après une sévère défoliation (Kébé, 2005). Toutes ces perturbations ont pour conséquence la chute rapide de la production (Mossu, 1990). Les pertes post-récoltes causées par cette maladie sont comprises entre 30 à $40 \%$ (Dzahini-Obiatey et al., 2010). II n'existe actuellement aucun traitement (Kébé et al., 2016) contre cette maladie et les seules méthodes adoptées sont l'arrachage des cacaoyers malades et la replantation avec des variétés résistantes ou tolérantes. La méthode de lutte qui préconise que l'arrachage est difficilement acceptées par les producteurs car les superficies détruites sont trop importantes et influencent négativement la situation socio-économique des producteurs. Face à cette situation des solutions alternatives doivent être trouvées à l'arrachage des cacaoyers malades. En effet, ces dernières années, une catégorie de produits et substances s'est développée au sein du marché des intrants agricoles « Les Biostimulants ». Les Biostimulants apportent des solutions en matière de fertilisation et souvent de protection des cultures (EBIC, 2014). Ils contiennent des substances ou des microorganismes qui ont pour fonction de stimuler les processus naturels pour accroître l'absorption, l'efficience des nutriments et la tolérance aux stress abiotiques et biotiques représentés par les maladies et les ravageurs. Les biostimulants augmentent aussi la qualité des récoltes et préservent la santé du sol, de l'environnement et de l'Homme (EBIC, 2014). C'est ce qui justifie cette étude dont le but est d'étudier l'effet du biostimulant Banzaï $\mathrm{TM}$ dans la régénération de la couverture foliaire des arbres de cacaoyers atteints de la maladie du "Swollen shoot". Plus spécifiquement il sera question d'évaluer l'effet du Biostimulant BanzaïTM dans la régénération de la couverture foliaire des cacaoyers apparemment sains et des cacaoyers atteints de la maladie du "Swollen shoot".
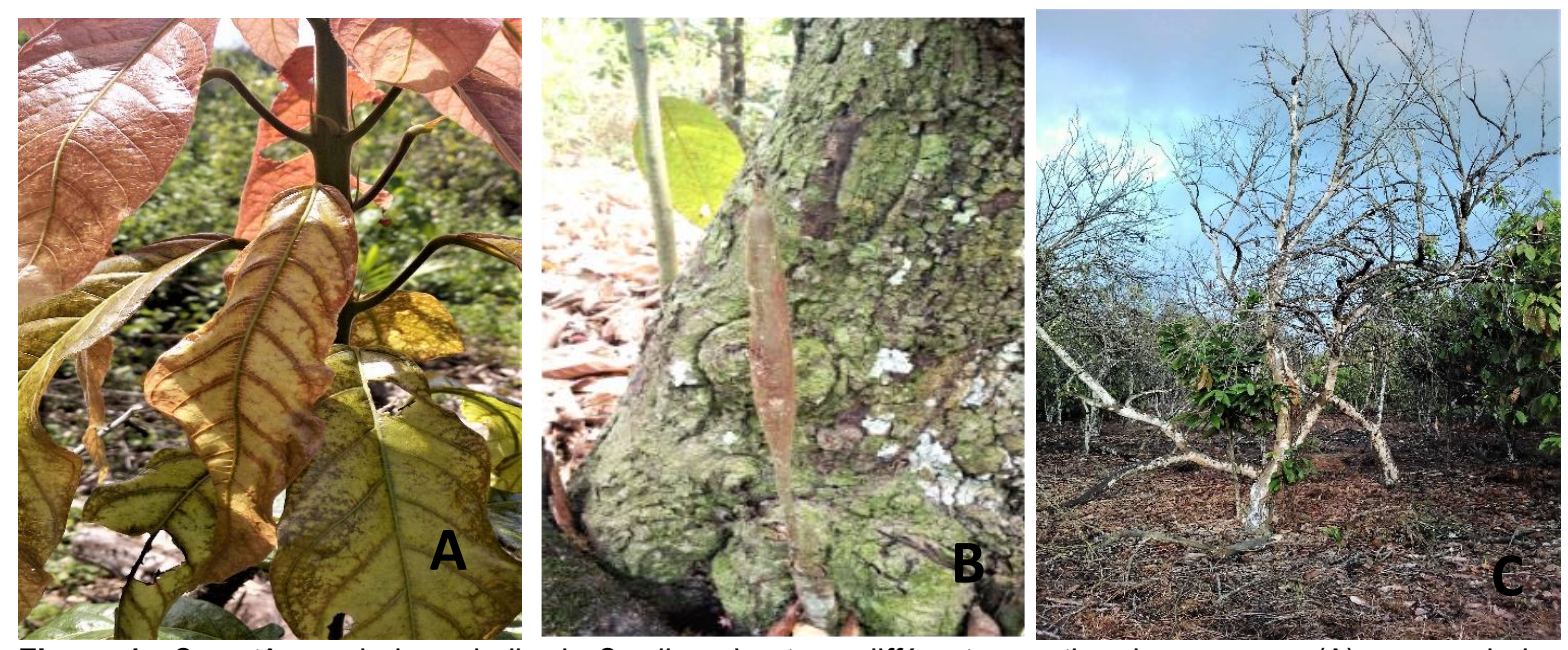

Figure 1: Symptômes de la maladie du Swollen shoot sur différentes parties du cacaoyer. (A) rougeur le long des nervures sur jeunes feuilles, (B) gonflement apical sur rameau orthotrope, (C) dégâts du Swollen shoot Virus dans les plantations paysannes avec des arbres malades présentant une défoliation complète (Photos prises par Oro, 2017).

\section{MATÉRIEL ET MÉTHODE}

Zone d'étude: Cette étude a été réalisée à Petit Bondoukou (Figure 2) situé à Méagui (département de Soubré) au Sud-Ouest de la Côte d'Ivoire, principale

zone de production du cacao avec environ 300.000 tonnes, soit près de $20 \%$ de la production nationale lors de la campagne 2013-2014 (AIP, 2015). Petit 
Bondoukou est un village situé à 51 kilomètres de Soubré. Ce village est inclus dans la région de la Nawa qui constitue aujourd'hui la principale zone de production du cacao en Côte d'Ivoire. Le site d'étude est caractérisé par un climat tropical humide avec une pluviométrie moyenne de $1485 \mathrm{~mm}$ par an, et une température moyenne de $25,8{ }^{\circ} \mathrm{C}$ par an. La végétation et le sol sont caractérisés par des forêts denses, humides et un sol profond, perméable et bien drainé qui permet une activité anthropique liée à l'agriculture (N'go et al., 2012). Cette végétation fait place aujourd'hui à des lambeaux de forêts et d'immenses plantations de cultures pérennes traditionnelles ou industrielles (AIP, 2015).
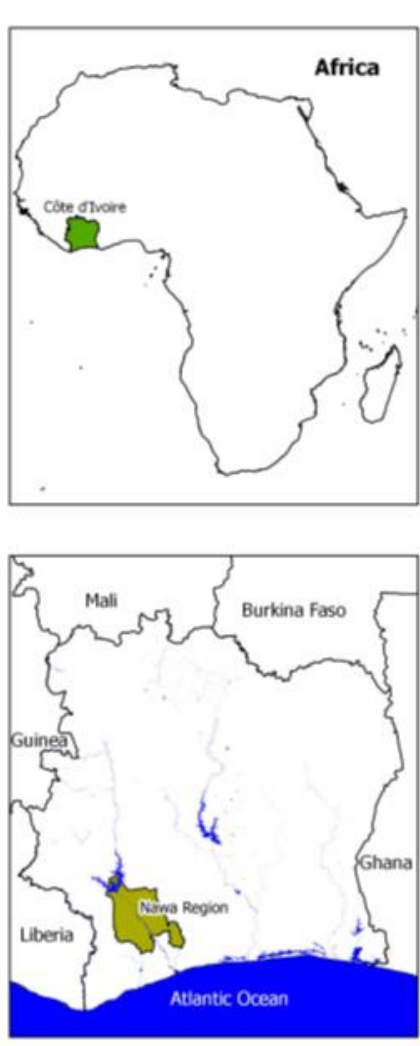

Côte d'Ivoire

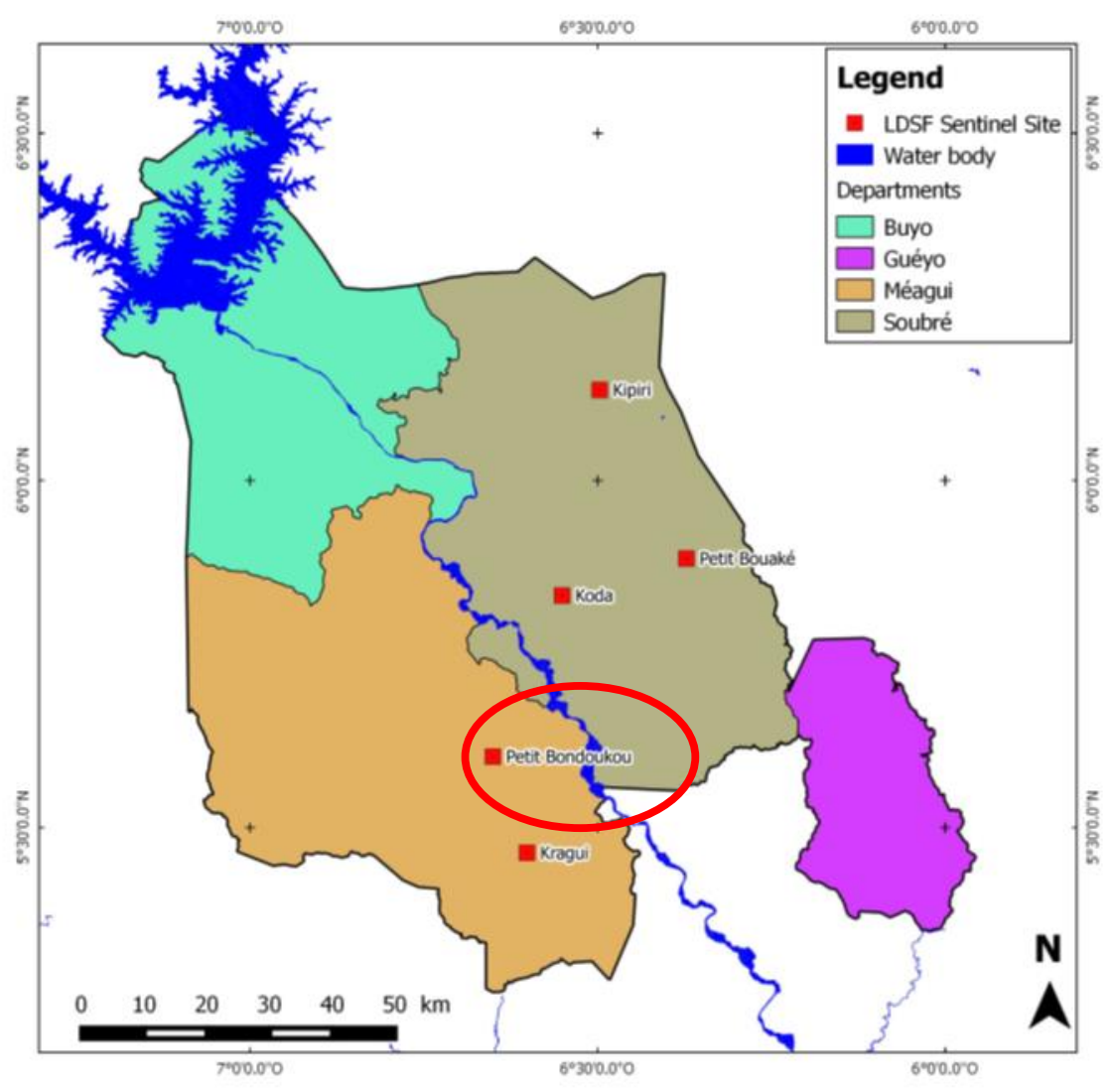

Figure 2 : Carte de la région de Nawa mettant en relief le site de Petit Bondoukou (Diby et al., 2014)

Dispositif expérimental: Le dispositif expérimental (Figure 3) est un bloc simple constitué de quatre (04) sous parcelles notées T0, T1, T2 et T3. Chaque parcelle expérimentale est composée de vingt (20) cacaoyers dont dix (10) arbres apparemment sains et 10 arbres malades. T0 représente le témoin sans application de Banzaï ${ }^{\mathrm{TM}}$ et sans apport d'engrais. T1 est la parcelle qui représente une application de Banzaï ${ }^{\mathrm{TM}}$ à dose normale et sans engrais. T2 est une parcelle représentant une application de BanzaïTM à double dose et sans un apport d'engrais et T3 représente une application de Banzaï TM à dose normale et avec apport d'engrais. Ce dispositif est répété 4 fois sur le site de Petit Bondoukou. 


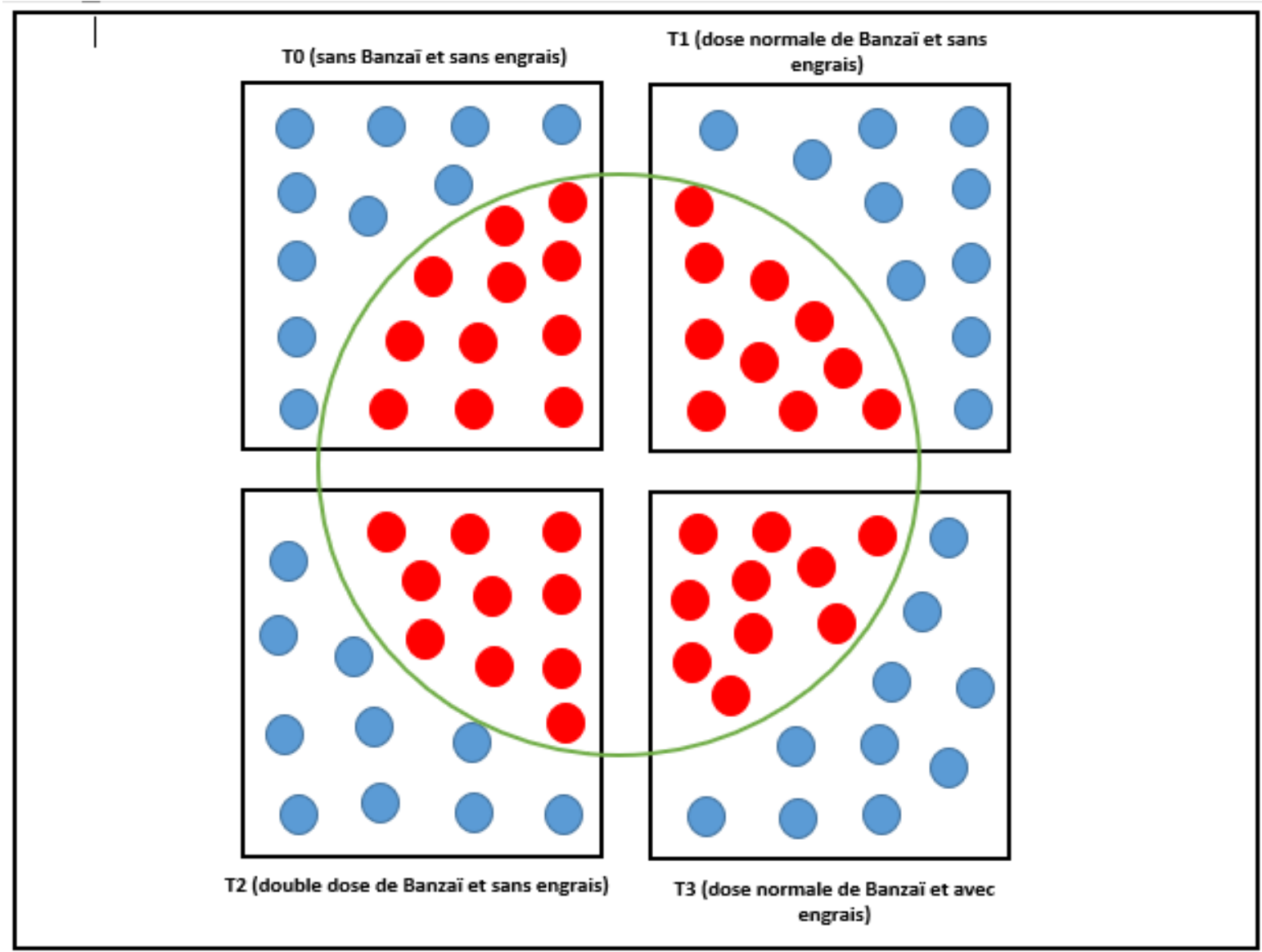

Arbre malade

Arbre apparemment sain

Limite du foyer d'infection

.Figure 3 : Dispositif expérimental avec les différentes parcelles élémentaires autour du foyer d'infection de CSSV.

Conduite de l'essai : La mise en place de l'essai a commencé par une enquête prospective qui a consisté d'abord à rechercher et choisir les sites d'expérimentation, puis à identifier les parcelles appropriées à l'étude. Les sites retenus ont été caractérisés par des parcelles cacaoyères avec présence de jeunes foyers d'infection contenant des arbres atteints de la maladie du "Swollen shoot", mais encore en production et des arbres apparemment sains. Chaque parcelle élémentaire a une superficie d'environ $300 \mathrm{~m}^{2}$ et contient 20 arbres dont 10 arbres malades et 10 arbres apparemment sains. Ces parcelles ont été délimitées dans le sens de progression du foyer d'infection. Le matériel végétal a été constitué de l'ensemble des cacaoyers tests qui ont été choisis dans les parcelles élémentaires déjà délimitées. Le matériel technique utilisé pour la mise en place de l'essai a été constitué de pots de peinture rouge et des étiquettes pour marquer les arbres tests, de rubans de plusieurs couleurs pour marquer les limites de chaque bloc et de chaque parcelle élémentaire, d'un atomiseur pour l'épandage du biostimulant Banzaï TM , d'un décamètre pour mesurer les limites des blocs et les limites des parcelles et un appareil photo pour des prises de vue. Les produits de traitement ont été constitués d'engrais SUPERCAO de formulation $0-23-19+10 \mathrm{CaO}+5 \mathrm{MgO}+6,5 \mathrm{~S}+0,7 \mathrm{Zn}$ qui a été appliqué sur certaines parcelles élémentaires, ensuite du biostimulant Banzaï ${ }^{\mathrm{TM}}$ avec une dose indicatrice de $800 \mathrm{ml} /$ ha qui a été appliquée sur l'ensemble des parcelles élémentaires à l'exception des parcelles témoins. En effet, l'engrais SUPERCAO a été appliqué deux fois c'est-à-dire une application lors de l'installation du dispositif en Août 2017 et l'autre application en Septembre 2017 (uniquement sur la parcelle élémentaire T3), en respectant les prescriptions et recommandations usuelles c'est-à-dire $200 \mathrm{~g} / \mathrm{arbre}$. L'épandage de l'engrais a été réalisé dans le périmètre de $50 \mathrm{~cm}$ de rayon autour de chaque arbre test et a été recouvert de la terre et de feuilles mortes. 
Par contre, l'application du biostimulant Banzaii TM a été fait sur la base de la dose par hectare (800 ml/ha), en considérant qu'un hectare de cacaoyers contient 1333 arbres de cacaoyers, il a été déduit la dose pour 20 pieds de cacaoyers ( $12 \mathrm{ml}$ pour 3 litres d'eau). La dose double (24 ml pour 3 litres d'eau) a été donc déduite de la dose simple. Le biostimulant Banzaï TM a été appliqué sur les deux faces du cacaoyer depuis le feuillage jusqu'au collet de l'arbre en passant par le tronc. Ainsi la dose normale de Banzaï TM a été appliquée sur les arbres tests des parcelles $\mathrm{T} 1$ et $\mathrm{T} 3$, tandis que la double dose a été appliquée sur les arbres tests de la parcelle $\mathrm{T} 2$.

Observations : Les observations en générale se sont réalisées chaque mois pendant six (06) mois (Août à Janvier) après l'épandage du produit Banzaï TM. Ces observations ont porté sur le taux de couverture foliaire aussi bien pour le lot d'arbres malades que pour le lot d'arbres apparemment sains. Les données collectées ont été consignées dans une fiche d'enquête.

Collecte de données: Le taux de couverture foliaire est une variable qualitative à trois modalités : le taux de couverture faible, le taux de couverture moyen et le taux de couverture fort. Le taux de couverture faible a été caractérisé par un arbre qui présente un aspect chétif et un faible ombrage. Les arbres à couverture faible sont moins productifs et ont tendance à perdre leurs feuilles tout au long de la saison. Par contre le

\section{RÉSULTATS}

Effet du Biostimulant Banzaï TM sur l'évolution de la couverture foliaire des arbres de cacaoyer apparemment sains : La Figure 4 présente l'évolution de la couverture foliaire par mois et par traitement. Dans l'ensemble, l'évolution de la couverture foliaire est sensiblement la même d'un traitement à un autre. La couverture foliaire qui était largement dominée par le taux moyen au début des observations a évolué au profit du taux de couverture fort excepté le mois de Janvier, où le taux de couverture foliaire des traitements $\mathrm{T} 2$ et du témoin T0 est resté relativement faible pour la quasi-totalité de leurs arbres tests. La simple dose de Banzaï TM représentée par le traitement T1 influence fortement la variation du taux de couverture foliaire comparativement à la double dose de Banzaï TM (T2) chez les arbres apparemment sains. Les résultats du tableau croisé montrent une différence du taux de la couverture foliaire entre le témoin T0 et les traitements. Le témoin a le plus grand nombre taux de couverture fort a été représenté par des arbres avec un fort ombrage et une couronne couvrante. Ces arbres sont également apparemment sains et très productifs. A l'intermédiaire du taux de couverture faible et du taux de couverture fort, se situe les arbres à taux de couverture moyenne.

Analyse des données : Les données ont fait l'objet d'abord d'une analyse descriptive sous Excel 2013, puis d'une analyse comparative à l'aide du logiciel IBM SPSS Statistics version 20. L'analyse descriptive des données a consisté à évaluer les proportions de chaque modalité (faible, moyenne ou forte) de couverture foliaire par mois d'observation, puis un histogramme a été représenté pour chaque traitement pour comprendre l'évolution de la couverture d'une période à une autre pendant les six mois d'observation. Des graphiques et des tableaux d'effectifs ont été réalisés pour visualiser les probables liens entre les traitements et les variables du taux de couverture. Les hypothèses émises à partir des graphiques et tableaux ont été confirmés ou infirmés par le test statistique de Khi-deux. Ce test a été choisi parce qu'il s'agissait de comparer deux variables qualitatives. Lorsqu'une différence a été trouvée à partir du test de Khi-deux, une classification des traitements a été faite pour déterminer le traitement qui permet d'avoir une bonne couverture foliaire.

d'arbres tests avec un taux de couverture foliaire faible comparativement aux arbres tests des parcelles traitées (T1, T2 et T3). Ainsi la différence de taux de couverture faible entre T0 et T1 est de $7 \%$, celle entre T0 et T3 est de $9 \%$; et celle entre T0 et T2 est de $6 \%$. Le taux de forte couverture foliaire pour les traitements $\mathrm{T} 1$ et T3 sont respectivement de $10 \%$ et $9 \%$ supérieures à celui du traitement T0 tandis que le taux de forte couverture foliaire du traitement T2 est inférieur de $2 \%$ à celui du traitement TO. Le témoin TO et les traitements $\mathrm{T} 1$ et $\mathrm{T} 3$ ont des taux de couverture foliaire moyen quasiment similaires (environ 42\%). Le traitement T2 à un taux de couverture fort supérieure à celui du traitement T0 de 9\% (Tableau 1). Le test statistique de khi-deux révèle un lien hautement significatif ( $p$-value $=0.008<0.05)($ Tableau 2$)$ entre la couverture foliaire et les traitements. Cela signifie que le biostimulant Banzaï TM améliore la couverture foliaire des arbres apparemment sains. 


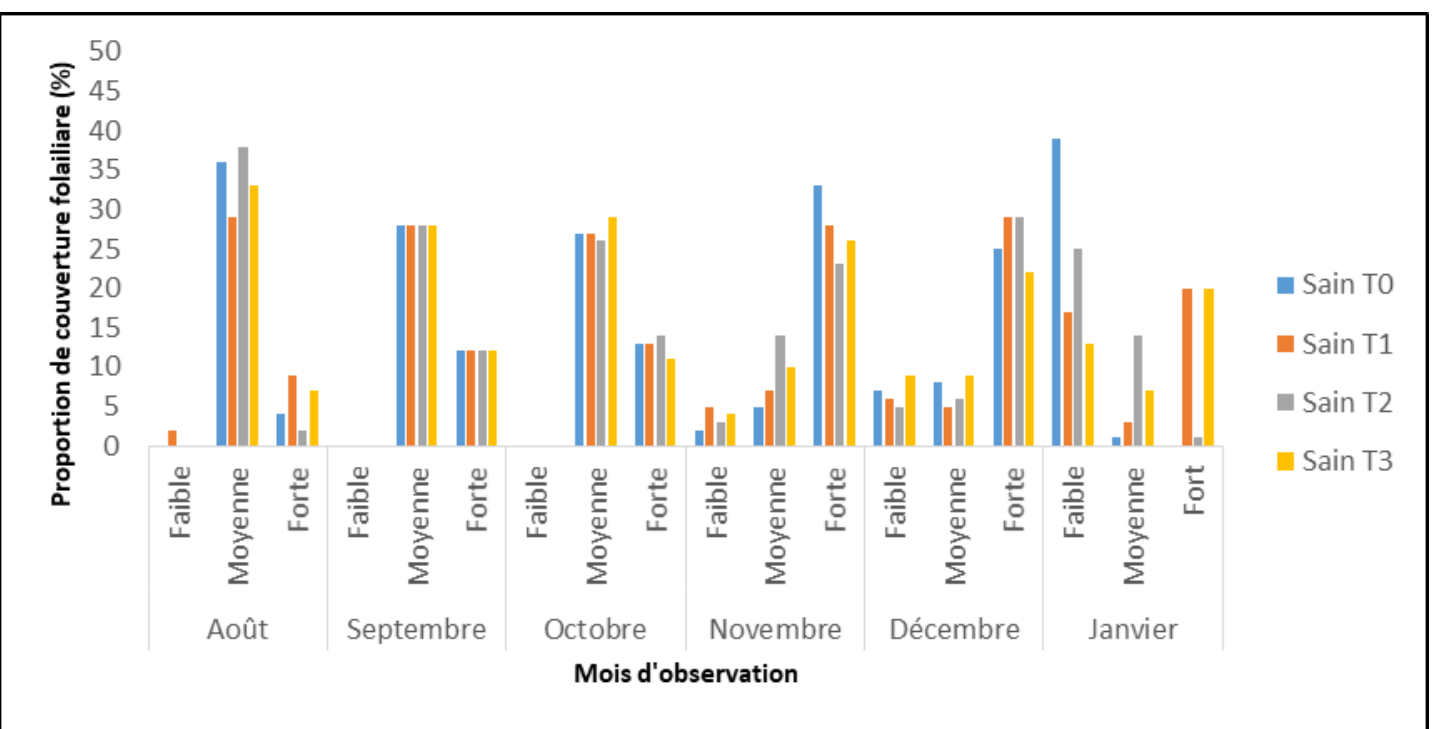

Figure 4 : Evolution du taux de couverture foliaire des arbres apparemment sains par traitement et par mois d'observation

Tableau 1 : Tableau d'effectif des taux de couverture foliaire en fonction des traitements chez les arbres sains

\begin{tabular}{ccccc}
\hline \multicolumn{5}{c}{ Traitements } \\
\hline Couverture foliaire & T0 & T1 & T2 & T3 \\
Faible & $20 \%$ & $13 \%$ & $14 \%$ & $11 \%$ \\
Forte & $36 \%$ & $46 \%$ & $34 \%$ & $41 \%$ \\
Moyenne & $44 \%$ & $41 \%$ & $53 \%$ & $48 \%$ \\
\hline
\end{tabular}

Tableau 2 : Résultat du test de khi-deux comparant la couverture foliaire aux différents traitements chez les arbres sains

\begin{tabular}{|c|c|c|c|c|}
\hline \multicolumn{5}{|c|}{ Tests du Khi-deux } \\
\hline & Valeur & ddl & p-value & alpha \\
\hline Khi-deux de Pearson & 17,474 & 6 & 0,008 & 0,05 \\
\hline Rapport de vraisemblance & 12,592 & 6 & 0,008 & 0,05 \\
\hline Nombre d'observations valides & 960 & & & \\
\hline
\end{tabular}

Effet du Biostimulant Banzaï TM sur l'évolution de la couverture foliaire des arbres malades : La Figure 5 présente l'évolution de la couverture foliaire par mois et par traitement. Au début des observations (Août), dans tous les traitements y compris le témoin, la majeure partie des arbres tests avaient une couverture foliaire moyenne. En Septembre et en Octobre, la couverture foliaire moyenne diminue au profit de la couverture foliaire forte en particulier sur les parcelles traitées T1, T2 et T3. Cette tendance a peu évolué en Novembre et en Décembre. Le mois de Janvier a été marqué par une grande défoliation des arbres des traitements TO et
T2 tandis que les arbres des traitements T1 et T3 ont eu la majeure partie de leurs arbres tests passée à une forte couverture foliaire. La simple dose de Banzaï ${ }^{\mathrm{TM}}$ avec apport d'engrais (T1) et la simple dose de Banzaï $\mathrm{TM}$ sans engrais (T3) favorisent un bon développement de la couverture foliaire des arbres tests atteints de la maladie du "Swollen shoot". Les résultats du tableau croisé montrent que les traitements T1, T2 et T3 ont des meilleures couvertures foliaires comparé à celui du traitement T0. Le taux de couverture foliaire faible des traitements T1, T2 et T3 sont inférieurs à celui de T0. Ainsi la différence de taux de couverture faible entre TO 
et T1 est de 13\%. Celle entre T2 et T0 est de $8 \%$ et celle de T0 et T3 est de $8 \%$. Tous les traitements T0, $\mathrm{T} 1, \mathrm{~T} 2$ et $\mathrm{T} 3$ ont des taux de couvertures foliaires moyens sensiblement similaire autour de $38 \%$ environ. Les taux de fortes couvertures foliaires des traitements $\mathrm{T} 1, \mathrm{~T} 2$ et $\mathrm{T} 3$ sont supérieurs à celui du témoin T0 de
12\% (Tableau 3). Le test statistique de khi-deux a aussi révélé qu'il existait un lien (Tableau 4) entre le traitement et la couverture foliaire ( $p$-value $<0,05)$. Le biostimulant Banzaï TM a eu un effet significatif sur la stimulation de la couverture foliaire des arbres apparemment malades.

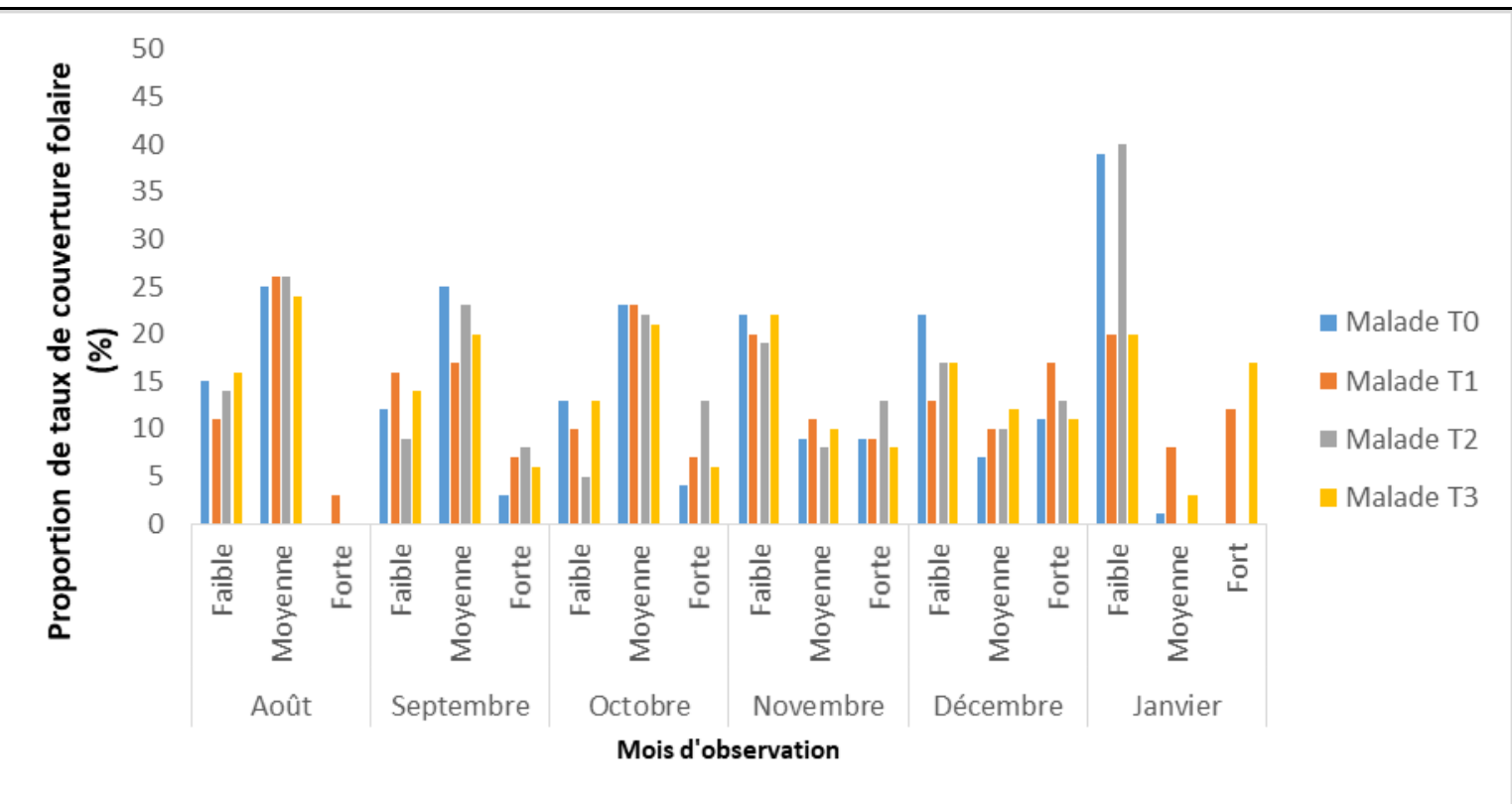

Figure 5 : Evolution du taux de couverture foliaire des arbres malades par traitement et par mois d'observation

Tableau 3 : Taux de chaque type du couvert foliaire en fonction des traitements chez les arbres malades

\section{Traitements}

\begin{tabular}{ccccc} 
Couverture foliaire & T0 & T1 & T2 & T3 \\
Faible & $51 \%$ & $38 \%$ & $43 \%$ & $43 \%$ \\
Forte & $11 \%$ & $23 \%$ & $20 \%$ & $20 \%$ \\
Moyenne & $38 \%$ & $40 \%$ & $37 \%$ & $38 \%$ \\
\hline
\end{tabular}

Tableau 4 : Test de khi-deux comparant de la couverture foliaire aux différents traitements chez les arbres malades

\begin{tabular}{|c|c|c|c|c|}
\hline \multicolumn{5}{|c|}{ Test du Khi-deux } \\
\hline & Valeur & ddl & $p$-value & Alpha \\
\hline Khi-deux de Pearson & 15,401 & 6 & 017 & 0,05 \\
\hline Rapport de vraisemblance & 12,592 & 6 & 017 & 0,05 \\
\hline Nombre d'observations valides & 960 & & & \\
\hline
\end{tabular}

\section{DISCUSSION}

Chez les arbres sains comme chez les arbres malades, les résultats statistiques du test de khi-deux montrent qu'il existe un lien significatif entre le traitement et le développement de la couverture foliaire. Chez les arbres tests apparemment sains comme chez les arbres malades, l'application du produit biostimulant Banzaï quel que soit la dose et indépendamment de l'apport ou non d'engrais permet d'améliorer la couverture foliaire. En Effet le biostimulant Banzaï TM contient des éléments essentiels à la croissance et au 
développement de la plante. Ce résultat a été obtenu par Faessel et al. (2014). En effet ces chercheurs ont découvert que le Trichoderma harzianum (Trianum) sur des Zinnias permet un bon développement racinaire, foliaire et une bonne masse de la matière fraîche. La présence du virus du "Swollen shoot" une fois dans la plante provoque des perturbations physiologiques et morphologiques qui sont susceptibles de ralentir la croissance de l'arbre (Oro, 2011). En effet, selon l'EBIC (2014), les biostimulants contiennent des substances ou des microorganismes qui ont pour fonction de stimuler les processus naturels pour accroitre l'absorption et l'efficience des nutriments, la tolérance aux stress abiotiques ou biotiques et la qualité des récoltes lorsqu'ils sont appliqués aux plantes ou à la rhizosphère indépendamment du contenu en nutriments du biostimulant. Yakhin et al. (2017) et Faessel et al. (2014) ont orienté le mode d'action de ces biostimulants vers la stimulation de la germination, de la croissance racinaire, de la mise en place et de la croissance des plantes, de la photosynthèse, de l'absorption des nutriments du sol (Azote, Phosphore),

\section{CONCLUSION ET PERSPECTIVES}

Au terme de cette étude, il ressort que l'application du biostimulant Banzaï TM a eu un impact significatif sur l'amélioration du développement foliaire aussi bien chez les arbres apparemment sains que chez les arbres malades. II serait donc recommandable aux producteurs d'utiliser la simple dose de Banzaï ${ }^{\mathrm{TM}}$ sans application d'engrais pour accroitre la vigueur des de la résistance au stress biotique, du métabolisme de $\mathrm{N}$ et $\mathrm{P}$ du sol, de l'activité microbienne des sols et aussi la réduction ou l'amélioration des effets négatifs des facteurs de stress abiotiques (sécheresse, chaleur, froid, salinité, oxydation, stress mécanique ou chimique). Ces approches définitionnelles et les modes d'action proposés par ces auteurs ont été observés lors de cette présente étude où le couvert végétal des cacaoyers atteints de la maladie du "Swollen shoot" a été régénéré de près de $14 \%$ par le biostimulant Banzaï ${ }^{T M}$. En effet une plante déficitaire d'éléments nutritifs présente toujours un aspect chétif et perd ces feuilles réduisant ainsi l'activité photosynthétique de celle-ci. Ces résultats obtenus dans le cadre de cette étude stipulent donc que le biostimulant Banzaï ${ }^{\mathrm{TM}}$ a la capacité de stimuler le processus d'absorption des nutriments des cacaoyers déjà affaiblis par la maladie du "Swollen shoot", améliorant ainsi le taux de couverture foliaire. Les résultats issus de cette étude pourraient être utilisés par la recherche scientifique pour améliorer les méthodes lutte contre la maladie du "Swollen shoot".

cacaoyers aussi bien malades qu'apparemment sains dans le but d'optimiser la production dans le contexte de la maladie du "Swollen shoot". En perspective, il importe d'étudier le mécanisme d'action du biostimulant Banzaï TM sur des arbres de cacaoyers atteints de la maladie du "Swollen shoot" afin de mieux apprécier l'effet des biostimulants sur des arbres malades.

\section{REMERCIEMENTS}

Nos remerciements vont à l'endroit du cabinet d'expertise agricole SAG Sarl pour la conduite des expérimentations et la collecte des données.

\section{RÉFÉRENCES BIBLIOGRAPHIQUES}

Agence Ivoirienne de Presse (AIP), 2015. Monographie du département de Soubré sud-ouest de la Côte d'Ivoire. Disponible sur: https://news.abidjan.net/h/527425.html consulté le 03/04/2018.

Alibert $H_{\text {., }}$ 1946. Note préliminaire sur une nouvelle maladie du cacaoyer le "Swollen shoot". Agronomie tropicale, paris, v.1, pp. $34-43$.

ARAG, 2017. Theobroma cacao L. groupe génétique "Guiana" Développement de la filière locale du Cacao guyanais Programme d'actions de l'association ARAG. 28p.

Conseil Café-Cacao (CCC). 2015. manuel technique de cacaoculture durable. $13 p$.
Diby L., Kouassi G., N'guessan M., Yao E., Oro F., Aynekulu E., Kassin E., Kouamé C., Coe R., Shepherd K, (2014). Cocoa Land Health Surveillance: An evidence-based approach to sustainable management of Cocoa landscapes in the Nawa region, South-West Côte d'Ivoire. Working Paper 193, $34 \mathrm{p}$.

Dufumier M, 2016. L'adaptation de la cacao-culture ivoirienne au dérèglement climatique : L'agroécologie pourrait-elle être une solution? Rapport de mission, Côte d'Ivoire, $16 p$

Dzahini O. H., Domfeh O, Amoah F M, 2010. Review: Over seventy years of a viral disease of cocoa 
in Ghana: From researchers' perspective. Afr. J. Agric. Res. 5 (7): 476-485.

EBIC (European Biostimulants Industry Council), 2014. Promoting the biostimulant industry and the role of plant biostimulants in making agriculture more sustainable. Disponible sur : http://www.biostimulants.eul

Faessel L., Gomy C., Nassr N., Tostivint C., Hipper C., Dechanteloup A, 2014. Produits de stimulation en agriculture visant à améliorer les fonctionnalités biologiques des sols et des plantes [archive]. Étude des connaissances disponibles et recommandations stratégiques, rapport d'étude réalisé par Bio by Deloitte et RITTMO Agro-environnement pour le ministère de l'Agriculture, de l'Agroalimentaire et de la Forêt, 148 pages

Journée Nationale du Cacao et de Chocolat (JNCC), 2016. Evolution de la filière café-cacao de 2012-2016. 44p.

Kebe I, 2005. Cacaoyère ivoirienne en danger, le "Swollen shoot" progresse. CNRA, lepoint2005.

Kébé B.I., N'guessan K.F., Assiri A.A., Tahi G.M., N'guessan W.P., Aka A.R., Kouakou K. 2016. Bien lutter contre la maladie du "Swollen shoot" du cacaoyer en Côte d'Ivoire. Fiche cacaoyer n04. CNRA 2016. 4p.

Kouakou K., Kébé B., Kouassi N, Anno AP., Aké S., Muller E., 2011. Impact de la maladie virale du "Swollen shoot" du cacaoyer sur la production de cacao en milieu paysan à Bazré (Côte d'Ivoire). Biosciences $43: 2947-2957 p$.

LE PAYSAN, 2012. Le cacao de $A$ à $Z$, Le paysan $\mathrm{n}^{\circ} 003,68 \mathrm{p}$.

Lot H., Djiekpor E., Jacquemond M, 1991. Characterization of the genome of cacao Swollen shoot virus. J Gen Virol 72 : 17351739.

Mossu G., 1990. Le cacaoyer. Le technicien d'agriculture tropicale. $\mathrm{N}^{\circ} 14, \mathrm{pp} .:$ 9-109.

Muller E. et Sackey S, 2005. Molecular variability analysis of five new complete cacao Swollen shoot virus genomic sequences. Arch Virol 150: 53-66.

N'go P.K., Azzaoui F.Z., Ahami A.O.T., Aboussaleh Y., Lachheb A., Hamrani A, 2012. Déterminants socioéconomiques, environnementaux et nutritionnels de l'échec scolaire : cas des enfants résidant en zone cacaoyère de Soubré (Côte d'lvoire). Antropo, 28, 63-70.
Ollennu L. A. A. Owusu G. K. et Thresh JM, 1989. "Spread of cocoa Swollen shoot virus to recent plantings in Ghana." in: crop protection 8: $n^{\circ} 4$ : p. 251-264.

Oro F Z, 2011. Analyse des dynamiques spatiales et épidémiologie moléculaire de la maladie du "Swollen shoot" du cacaoyer au Togo : étude de la diffusion à partir des systèmes d'information géographiques, Montpellier SupAgro. Thèse de doctorat école doctorale sibaghe, 262p.

Posnette A F, 1940. Transmission of Swollen shoot. Trop. Agriculture, trin. pp: 17-98.

Van I M, 1998. Plant Growth Stimulators Effects on Post-Transplant Growth and Flowering of Petunia and Impatiens Plugs. HorTechnology. 8(1): 45-47.

Yakhin O.I., Lubyanov A.A., Yakhin I.A., Brown PH, 2017. Biostimulants in Plant Science: A global Perspective. Frontiers in Plant Science 7, p.132. 